DOI: $10.3901 / J M E .2021 .15 .160$

\title{
基于摩擦自激振动升频效应的超低频 振动能量收集*
}

\author{
陈 伟 1,2 项载毓 1,2 钱泓桦 1,2 莫继良 ${ }^{1,2}$
}

(1. 西南交通大学机械工程学院 成都 610031;

2. 西南交通大学轨道交通运维技术与装备四川省重点实验室 成都 610031)

\begin{abstract}
摘要: 低频振动在环境中广泛存在, 针对目前低频振动能量收集效率低的问题, 提出将外界低频振动转化成摩擦系统的自激 振动, 实现低频振动到高频振动的升频转换, 以达到提高低频能量收集效率的目的。为此, 首先建立了集总参数模型, 从理 论上阐明低频振动能量收集方法的可行性, 并分析模拟了该模型压电电压与功率的输出特性; 其次分别设计了往复摩擦自激 振动能量收集试验和压电悬臂梁碰撞能量收集试验, 对比分析将低频振动转化为摩擦振动能否提高压电输出功率。试验与仿 真结果表明, 利用摩擦自激振动能够显著提升振动频率。摩擦自激振动电压幅值随着激励频率的增大而增大, 与碰撞电压相 比, 其电压幅值较低, 但是频率得到了大幅提升。由于频率的提升, 压电振子的能量输出大幅提升, 使得超低频振动能量收 集性能明显提高。
\end{abstract}

关键词: 能量回收; 升频转换; 超低频振动; 摩擦自激振动

中图分类号: TH117

\section{The Frequency-up-conversion Effect Driven by Friction-induced Vibration for Ultra-low Frequency Vibration Energy Harvesting}

\author{
CHEN Wei ${ }^{1,2}$ XIANG Zaiyu ${ }^{1,2}$ QIAN Honghua ${ }^{1,2}$ MO Jiliang ${ }^{1,2}$ \\ (1. School of Mechanical Engineering, Southwest Jiaotong University, Chengdu 610031; \\ 2. Technology and Equipment of Rail Transit Operation and Maintenance Key Laboratory of Sichuan \\ Province, Southwest Jiaotong University, Chengdu 610031)
}

\begin{abstract}
Low-frequency vibration energy is ubiquitous in surroundings. However, it is a consensus that obtaining a satisfactory efficient of energy harvesting at ultra-low ambient frequencies remains challenging. Therefore, a solution to this problem is proposed, which is to increase the working frequency by converting the low-frequency excitation into self-excited vibration of friction systems. Inspired by the idea, a lumped parameter model is developed to confirm the feasibility theoretically, and the characteristics of the output voltage and power is analyzed and simulated based on the numerical model. An energy harvesting experiment where the excitation comes from the vibration induced by reciprocating sliding friction is performed, for comparison, an impact-driven energy harvesting experiment is also conducted, to confirm the efficacy of improving the output power by converting the ultra-low-frequency excitation into friction-induced vibration of friction systems. Both the experimental and numerical results indicate that the working frequency can be tremendously improved via friction-induced vibration, and the amplitude of the voltage generated by the friction-induced vibration is positively correlated with the excitation frequency. Compared with the voltage generated by the impact, the voltage generated by the friction-induced vibration owns lower amplitude but much higher frequency. Thanks to the frequency-up-conversion effect, the output power of the energy harvester is improved significantly, which enhances the performance of the energy harvester at ultra-low ambient frequencies.
\end{abstract}

Key words: energy harvesting; frequency-up-conversion; ultra-low frequency vibration; friction-induced vibration

* 国家自然科学基金(51822508)和四川省科技计划(2020JDTD0012)资助

项目。20200903 收到初稿, 20201215 收到修改稿 


\section{0 前言}

近年来微机电系统与无线微型传感器的发展十 分迅速, 小型化与低功耗是其发展的主要趋势。目 前, 对这些器件的供能大多依赖于传统的化学电池, 考虑到电池寿命较短, 频繁地对电池进行更换或充 电有诸多不便, 并且会带来环境污染等问题, 因此 有必要发展出一套微型传感器自供能技术 ${ }^{[1]}$ 。鉴于 振动能量在环境中普遍存在, 一种简单而有效的环 境振动能量收集方法为这种微型传感器自供能技术 提供了可能, 近年来受到了广泛的关注 ${ }^{[2-5]}$ 。

在诸多的振动能量收集方法当中, 压电能量收 集以其较高的能量密度和简单实用的结构而备受关 注 $^{[4,6]}$ 。压电能量转化的原理是依靠悬臂梁振动时产 生的应变而产生电压, 由于其固有结构的特点, 悬 臂梁式的能量收集器固有频率较高, 因此共振频率 较高 ${ }^{[7]}$ 。然而, 自然环境中存在的振动普遍为低频 振动, 其特点是频率低、振动幅值大。例如, 人走 路的频率大致在 2 3 Hz 之间, 海浪波动以及高层 或大型建筑的振动频率大都集中在 $10 \mathrm{~Hz}$ 以下甚至 更低 ${ }^{[7-9]}$ 。压电悬臂梁对低频激励极不敏感, 其能量 回收效果极差。为了提高发电功率, 现阶段采取的 一般措施是在悬臂梁自由端施加质量块降低其固有 频率, 但这会使得系统刚度过低容易引起结构损 坏 ${ }^{[10]}$ 。因此, 近年来研究人员开始探究如何实现低 频振动到高频振动的升频转换以提高能量收集效 率 ${ }^{[7,9]}$ 。一般而言, 压电式振动能量收集器的产生功 率与其振动频率是呈正相关的, 低频率意味着低能 量密度。因此, 为提高振动能量收集效果, 需将低 频振动转化为高频振动以调和低频激励与压电悬臂 梁高频响应之间的矛盾 ${ }^{[11-13]}$ 。

摩擦自激振动是由两个相互接触的物体相对运 动时所产生的界面摩擦诱导的自激振动, 通常具有 较高频率的特点, 仅在低速 stick-slip 运动条件下会 具有一定的低频特性 ${ }^{[14]}$ 。此外, 摩擦自激振动频率 只与摩擦系统的固有属性有关(系统的刚度、质量 等), 与外界激励频率无关。摩擦自激振动是一种潜 在的压电能量回收激励源, 其能够使得压电振子避 免因外界激励频率变化而出现频率失匹。因此, 如 果能将摩擦自激振动引入到低频振动激励的工况环 境中, 将有望实现低频振动到高频振动的升频转换。 然而, 目前关于利用摩擦自激振动实现能量回收的 研究极少 ${ }^{[15-17]}$ 。

本文提出了一种利用摩擦自激振动实现升频转
换的能量收集方式，并建立了含悬臂梁结构的集总 参数数值模型以从理论上证明所提方法的可行性。 基于建立的数值模型, 首先探讨了摩擦系统的压电 电压与功率输出特性, 而后分析了在不同外界激励 频率下系统的输出电压。此外, 开展了摩擦自激振 动能量回收试验以验证理论计算结果, 并设计了碰 撞式能量回收试验作为对比, 比较了两种试验的输 出电压以及在最优电阻下的输出功率大小。研究结 果有望为微机电系统和无线小型传感器等低功耗器 件的供能提供新的思路和方法。

\section{1 理论模型与分析}

\section{1 数值模型}

本文建立了含悬臂梁结构的集总参数模型, 如 图 1 所示。其中, $M$ 是一质量块, 下面为一刚性传 输带, 做周期性的正弦往复运动, 代表外部的振动 激励; 质量块 $M$ 与传输带之间具有线性接触刚度 $K_{1}$ 与三次非线性接触刚度 $K_{\mathrm{nl}}$, 坚直运动方向还具 有阻尼 $C_{1}$; 质量块 $M$ 水平运动方向具有刚度 $K_{2}$ 与 阻尼 $C_{2}$, 同时在于水平方向成 $45^{\circ}$ 角的方向上有弹 簧 $K_{3}$, 起到耦合 $M$ 坚直方向运动与水平方向运动的 作用。 $M$ 在坚直方向受到压力 $F$ 的作用。 $m$ 代表压 电悬臂梁的等效质量, $K_{p}$ 与 $C_{p}$ 分别代表压电悬臂梁 的等效刚度与等效阻尼。当传输带运动时, 由于摩 擦力的作用, $M$ 会发生自激振动, 得益于 $M$ 的水平 振动, 压电悬臂梁 $m$ 会在水平方向上做受迫振动, 其运动自由度定义为 $x_{p}$, 此时压电材料将产生电压 $U$, 将振动能转化为电能。为了简单起见, 本模型 不考虑质量块的与传输带之间的黏着状态，只考虑 滑动状态。为了与实际情况相对应, 质量块 $M$ 远大 于压电悬臂梁质量 $m$, 对应的刚度阻尼也远大于悬 臂梁的等效刚度与阻尼。

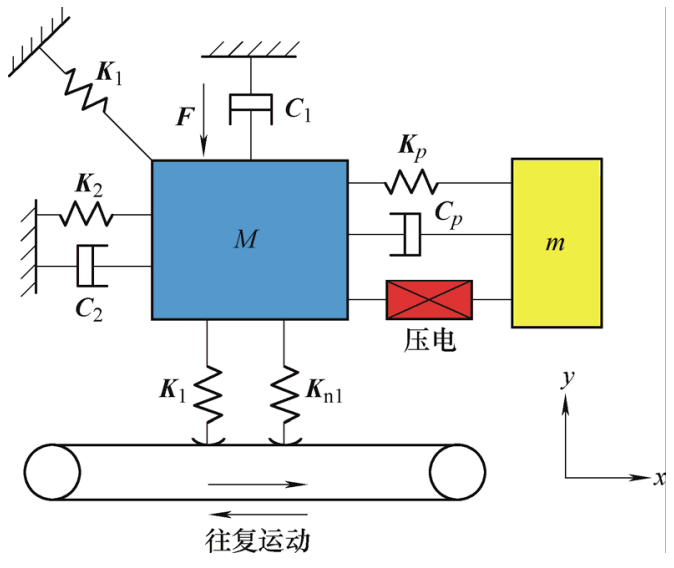

图 1 集总参数模型 


\section{2 动力学建模}

根据牛顿运动定律与基尔霍夫定律, 可得摩擦 系统的动力学方程与压电方程

$$
\left\{\begin{array}{l}
M \ddot{x}+\left(c_{2}+c_{p}\right) \dot{x}-c_{p} \dot{x}_{p}+\left(K_{2}+K_{p}+\frac{K_{3}}{2}\right) x+ \\
\left(\mu K_{1}-\frac{K_{3}}{2}\right) y-K_{p} x_{p}-\alpha U+\mu K_{n l} y^{3}=0 \\
M \ddot{y}+C_{1} \dot{y}+\left(K_{1}+\frac{K_{3}}{2}\right) y-\frac{K_{3}}{2} x+K_{n l} y^{3}=-F \\
m \ddot{z}+C_{p} \dot{z}+K_{p} z+\alpha U=-m \ddot{x} \\
\alpha \dot{z}-C \dot{U}-\frac{U}{R}=0 \\
z=x_{p}-x
\end{array}\right.
$$

式中, $\alpha$ 是力电耦合系数; $\mu$ 为摩擦因数; $C$ 为压电 陶瓷等效电容; $R$ 是外接电阻; $x$ 代表图 1 中质量块 $M$ 沿着坐标轴 $x$ 方向运动的位移; $y$ 代表质量块 $M$ 沿着坐标轴 $y$ 方向的位移; 其中 $x_{p}$ 代表压电悬臂梁 等效质量块 $m$ 沿着坐标轴 $x$ 方向的位移, 而 $z$ 是 $m$ 在 $x$ 轴方向上的位移与 $M$ 在 $x$ 方向上的位移之差, 即 $z=x_{p}-x$, 代表压电悬臂梁的变形量。传输带做正 弦往复运动, 其位移方程为

$$
y=Y \sin (\omega t)
$$

由于传输带运动速度的大小与方向时刻都在变 化, 因此质量块所受的摩擦力的方向会发生改变。 本文以质量块 $M$ 水平速度与传输带的速度之差来判 定摩擦力的方向, 摩擦系数的数学表达式如下

$$
\mu=\mu_{0} \operatorname{sgn}(\dot{y}-\dot{x})
$$

式中, $\mu_{0}$ 为 0.8 。这里需要说明的是, 建立本数值模 型的主要目的并不是为了定量地重现实验结果, 而 是为了说明利用摩擦自激振动可以实现升频转化并 提高超低频振动下的能量收集效率的基本特征，因 此本模型中的参数是根据一般经验所大致选取, 并 不代表后面实验中的真实参数。本数值模型中的参 数如表 1 所示。

表 1 数值模型参数

\begin{tabular}{cc||cc}
\hline 参数 & 数值 & 参数 & 数值 \\
\hline 刚度 $K_{1} /\left(\mathrm{N} \cdot \mathrm{m}^{-1}\right)$ & $2 \times 10^{7}$ & 阻尼 $C_{1} /\left(\mathrm{kg}^{-1} \mathrm{~s}^{-1}\right)$ & 600 \\
刚度 $K_{2} /\left(\mathrm{N} \cdot \mathrm{m}^{-1}\right)$ & $1 \times 10^{7}$ & 阻尼 $C_{2} /\left(\mathrm{kg}^{-1} \mathrm{~s}^{-1}\right)$ & 529.5 \\
接触刚度 $K_{\mathrm{n}} /\left(\mathrm{N} \cdot \mathrm{m}^{-3}\right)$ & $1 \times 10^{7}$ & 等效阻尼 $C_{p} /\left(\mathrm{kg}^{-1} \mathrm{~s}^{-1}\right)$ & 30 \\
等效刚度 $K_{\mathrm{p}} /\left(\mathrm{N} \cdot \mathrm{m}^{-1}\right)$ & $1 \times 10^{5}$ & 力 $F / \mathrm{N}$ & 100 \\
刚度 $K_{3} /\left(\mathrm{N} \cdot \mathrm{m}^{-1}\right)$ & $2 \times 10^{7}$ & 质量块 $M / \mathrm{kg}$ & 1 \\
等效电容 $C / \mathrm{F}$ & $2 \times 10^{-7}$ & 等效质量块 $m / \mathrm{kg}$ & 0.01 \\
电耦合系数 $\alpha /\left(\mathrm{N} \cdot \mathrm{V}^{-1}\right)$ & $1 \times 10^{-5}$ & 外接电阻 $R / \Omega$ & 1500 \\
\hline
\end{tabular}

\section{3 数值模拟}

基于建立的摩擦系统的动力学方程与压电方 程, 使用 MATLAB 中的 ode45 函数对其进行数值模 拟。首先将传输带的往复频率定为 $2 \mathrm{~Hz}$, 即相当于 外界激励频率为 $2 \mathrm{~Hz}$, 得到质量块的切向振动位移 和输出电压, 如图 $2 \mathrm{a}$ 和 $2 \mathrm{~b}$ 所示。结果表明, 当传 输带往复一个周期时, 系统会经历一次高频振动, 相应的也会输出一段高频的电压信号。由于往复摩 擦的特性，系统仅在往复运动的前半个行程中发生 摩擦自激振动 ${ }^{[18]}$, 因此电压信号也只在对应的半个 行程中产生。对数值模拟得到的电压信号进行功率 谱密度分析, 结果如图 2c 所示, 可以看出电压信号 的主频为 $820 \mathrm{~Hz}$, 这个频率远远高于激振频率 $2 \mathrm{~Hz}$, 因此振动频率得到了极大的提升。

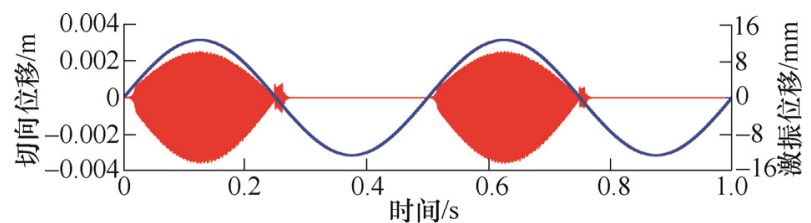

(a)

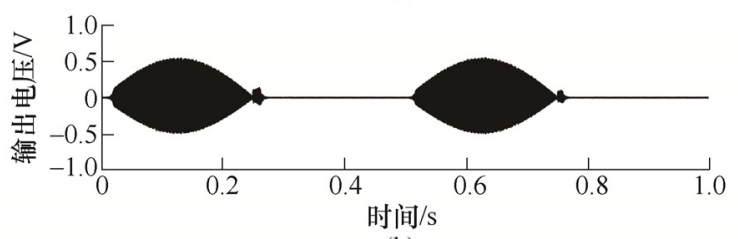

(b)

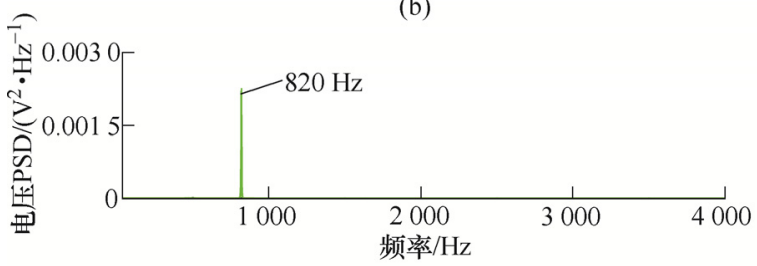

(c)

图 2 质量块的切向位移与压电输出电压以及 电压功率谱密度分析

图 3 所示为摩擦系统在不同外接电阻下的位 移一速度相图, 从图 3 中可以看出在不同的外接电 阻下摩擦系统的相图没有出现明显差异，即外接电 阻并不改变摩擦系统的运动状态。

$z$ 作为压电方程的输入, 其不随外接电阻 $R$ 的 改变而改变, 因此可以将压电方程从方程组(1)中解 耦出来, 单独分析压电方程。为了简单起见, 令 $z=A \sin (\omega t)$, 解出电压响应

$$
U=U_{0} \exp \left(\frac{-t}{R C}\right)+\frac{A \alpha}{C} \sqrt{1-\frac{1}{1+(R C \omega)^{2}}} \sin (\omega t+\varphi)
$$

式中, 相位 $\varphi$ 有如下关系式

$$
\tan \varphi=\frac{1}{R C \omega}
$$



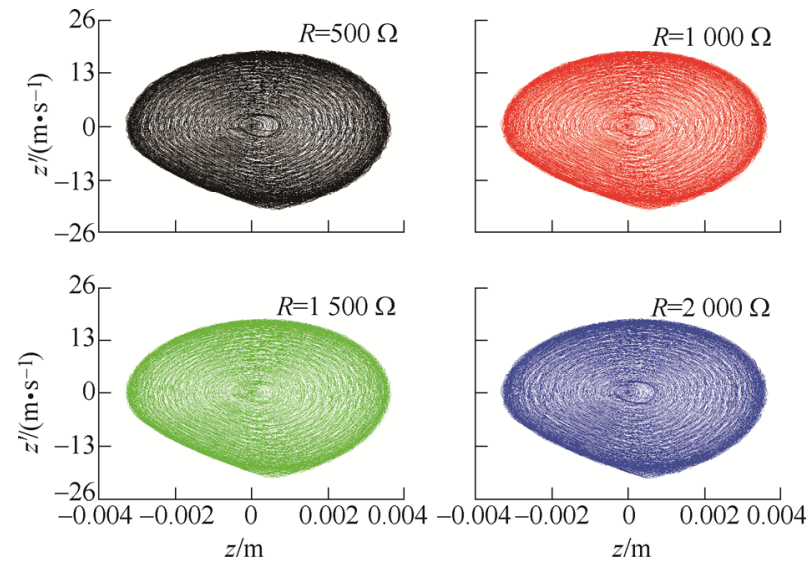

图 3 不同电阻下系统的运动相图

$U_{0}$ 项是压电齐次方程的通解, 由于压电电容的 时间常数 $R C$ 很小, 方程式(4)中的第一项会在很短 时间内按指数衰减至接近于零, 电压的稳态响应为 方程中的第二项, 从中不难发现电压 $U$ 的频率与激 振频率相同, 且电压幅值随着电阻 $R$ 的增大而增大, 当 $R$ 增大到一定极限时, 电压幅值趋于最大值: $A \alpha / C$ 。针对方程组(1)中的电阻 $R$ 值取若干数值, 得 到不同电阻下的电压幅值, 并与方程式(4)的结果进 行比较, 结果如图 4 所示。可以看出理论推导与数 值仿真结果差异很小, 也证实了上面的推导, 说明 该系统具有上述的电压-电阻关系是由于外接电阻 不改变系统的动力学特性所决定的, 这与 WANG 等 ${ }^{[15]}$ 的试验结果相符。

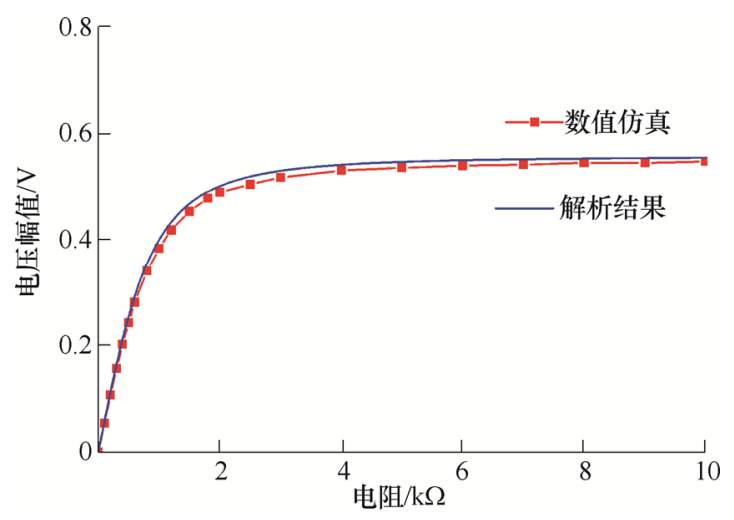

图 4 电压幅值随外接电阻的变化曲线

一般地, 压电陶瓷相当于电容, 当外界振动使 得压电陶瓷产生交变电流后, 其内部阻抗为

$$
Z_{p}=\frac{1}{2 \pi f C i}
$$

式中, $f$ 为压电电压频率, 当外接负载为 $Z$ 时, 负载 功率为

$$
P=\frac{U_{m}^{2}}{2\left|Z+Z_{p}\right|^{2}} \operatorname{Re}(Z)
$$

式中, $U_{m}$ 是压电开路电压幅值。当外接电阻阻值等 于压电陶瓷内阻时, 系统输出功率最大

$$
P=\frac{U_{m}^{2}}{8\left|Z_{p}\right|}
$$

数值模拟的电压频率为 $820 \mathrm{~Hz}$, 由式(6)可以得 出最优电阻 $R_{o p t}=970 \Omega$ 。由数值模拟(方程组 1)得到 的电阻功率曲线如图 5 所示, 可见系统输出功率随 着电阻的增大先增大后减小, 存在一个最优外接电 阻值使得输出功率达到最大, 最优电阻值与方程式 (6)的计算结果一致。

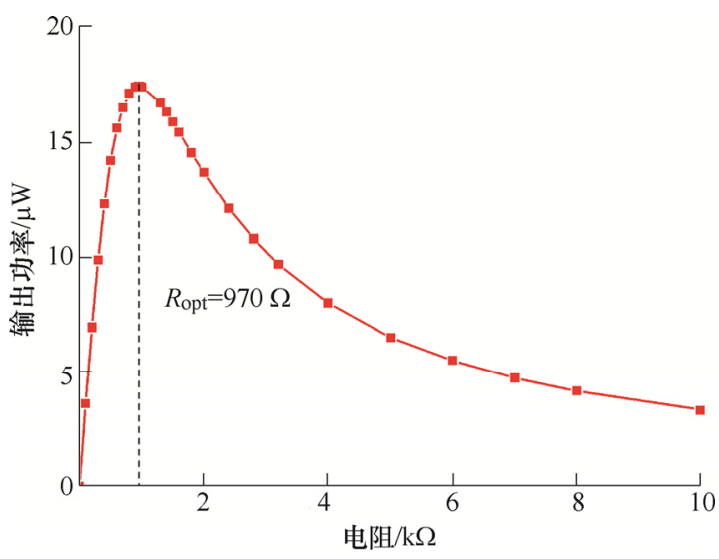

图 5 输出功率随电阻变化曲线

为了研究外界激励频率对压电电压的影响, 本 文计算了外界激励频率分别为 $1 \mathrm{~Hz} 、 2 \mathrm{~Hz} 、 3 \mathrm{~Hz}$ 和 $4 \mathrm{~Hz}$ 下摩擦系统的电压信号。其中，外界激励振动 的运动幅值均保持为 $10 \mathrm{~mm}$ 。经数值模拟计算可得 到摩擦系统的电压信号如图 6 所示。从图 6 中可以 看出输出电压高频振荡的次数与激励频率相等, 这 主要是由往复摩擦的振动特性所决定的。随着激励 频率的增加, 电压振荡的幅值随之增加, 其原因可 能是当往复激励频率增加时, 传输带往复运动的速 度也随之增加, 界面摩擦馈入系统的能量增加, 增
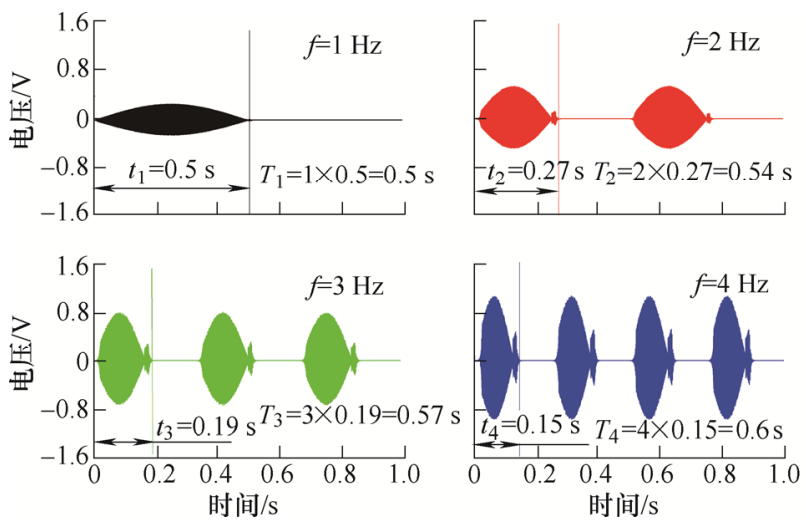

图 6 不同激振频率下的输出电压 
强了系统的自激振动, 导致了压电电压的振动幅 值增大。对电压信号进行 FFT 分析, 发现不同往 复频率下的电压信号的频率并未发生改变, 如 图 7 所示。此外, 当激振频率增大后, 电压单个 的振荡周期在缩短, 这是因为传输带往复运动的 幅值未变, 而往复频率增大, 导致传输带单个行 程所用的时间减少, 因此电压单个连续振荡周期 缩短。然而, 在单位时间内电压所经历的总的振 荡时间却并未减少, 反而在逐步上升, 因此电压 信号看上去更加 “密集”, 这也说明系统输出的 电能在增加。

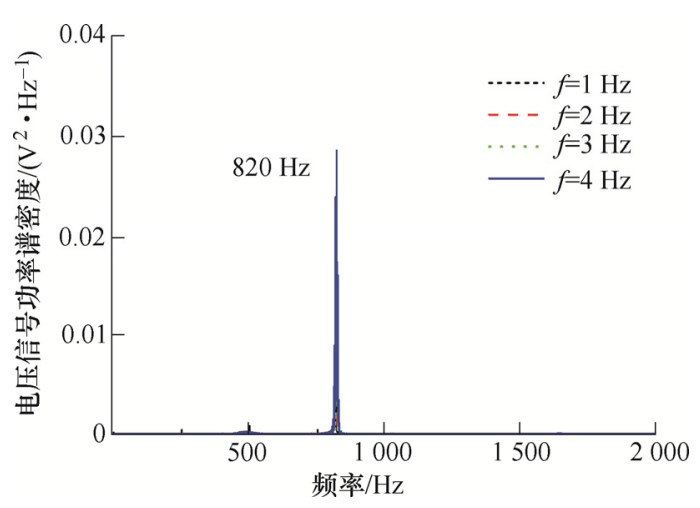

图 7 不同激振频率下的电压信号 PSD 分析

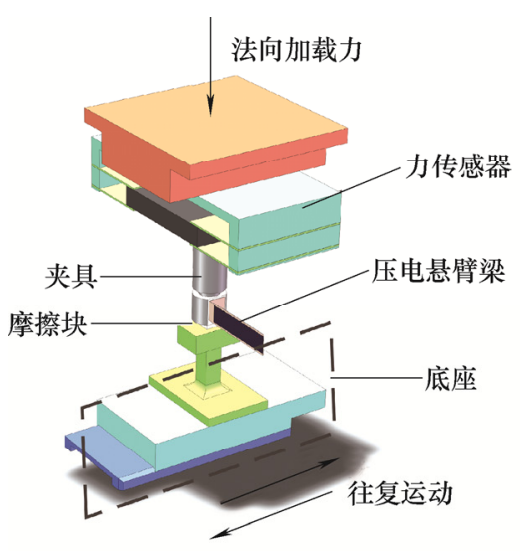

(a) 摩擦自激动振压电发电示意图

\section{2 试验测试}

\section{1 试验装置及方法介绍}

低频振动能量收集试验在 CETR UMT-3 试验机上 进行, 分别开展了摩擦自激振动能量回收试验与碰撞式 能量回收试验, 如图 8 所示。其中, 图 8a 展示的是摩 擦自激振动能量收集的示意图。整个装置分为上下两部 分, 上面部分由力传感器、夹具、压电悬臂梁组成, 压 电悬臂梁粘贴在夹具末端侧面, 夹具末端固定有摩擦 块, 上端与力传感器相连接; 下部分主要是底座, 由往 复电机驱动, 其上固定有摩擦材料。试验开始时, 先加 载法向力, 使得摩擦块与底座上的摩擦材料接触, 而后 启动往复电机带动底座做往复运动。由于接触界面之间 的摩擦会使系统产生摩擦自激振动, 使得压电悬臂梁变 形从而产生电压信号。作为对比, 也开展了传统的低频 振动能量收集试验, 即通过底座的往复运动将安装在其 上的悬臂梁与阻挡装置发生碰撞以产生较大变形, 从而 获取一定幅值的电压, 如图 $8 \mathrm{~b}$ 所示。毕竟, 如果不设 置碰撞环节, 仅底座带着压电悬臂梁做低频往复运动, 悬臂梁在这种低频激励下难以发生共振, 几乎没有电压 产生。两种装置的压电悬臂梁的尺寸均为 $40 \mathrm{~mm} \times$ $16 \mathrm{~mm} \times 0.3 \mathrm{~mm}$, 压电材料型号为 PZT- $5 \mathrm{H}$ 。

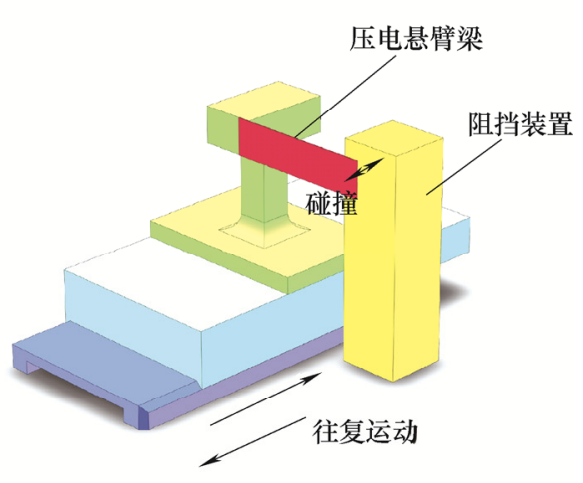

(b) 碰撞式压电发电示意图

图 8 两种能量回收方式装置示意图

\section{2 试验结果及分析讨论}

首先, 为了从试验上验证依靠摩擦自激振动提 升振动频率并实现低频振动能量收集的可行性, 将 图 $8 \mathrm{a}$ 中试验装置的底座的往复频率设定为 $2 \mathrm{~Hz}$, 底座的往复运动等效于外界低频的振动激励源, 往 复行程为 $10 \mathrm{~mm}$, 法向力设定为 $20 \mathrm{~N}$ 。使用加速度 传感器测量系统的振动, 并将压电陶瓷的电极接入 数据采集系统进行电压信号的采集。

图 9 所示为试验过程采集到的切向振动加速度
和电压信号, 以及电压信号的功率谱密度。结果表 明, 系统的振动加速度和电压信号与理论计算结果 相符。当底座在一秒钟之内往复两次时, 系统经历 了两次高频振动, 都在底座往复运动的前半个行程 中出现, 与此同时, 压电悬臂梁也对应地输出了两 段高频电压信号, 电压幅值为 $1 \mathrm{~V}$ 左右。对电压信 号做功率谱密度分析, 发现其主频为 $4600 \mathrm{~Hz}$ 。因 此, 通过摩擦自激振动有效地将激振频率从极低的 $2 \mathrm{~Hz}$ 大幅度提升到了 $4600 \mathrm{~Hz}$, 试验结果也证明了 
本文所提出的基于摩擦自激振动升频效应实现低频 振动能量收集的可行性。

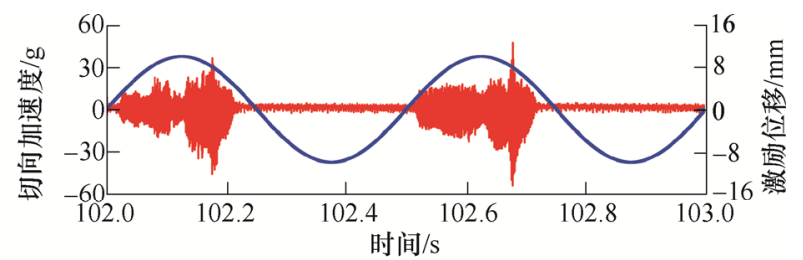

(a) 底座位移曲线与系统切向振动加速度

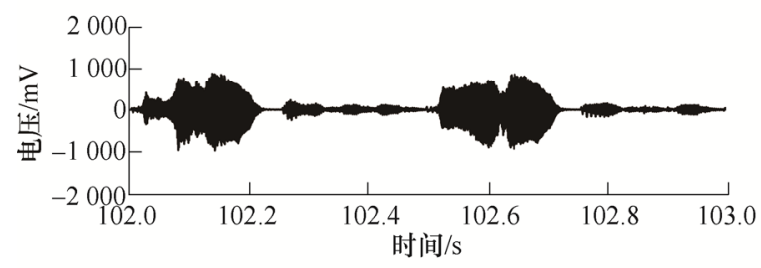

(b) 压电电压信号

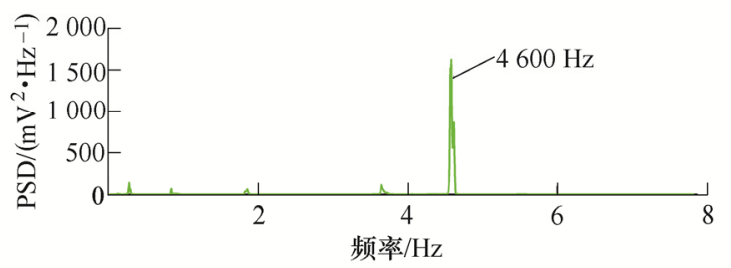

(c) 电压信号功率谱密分析

图 9 切向振动加速度和输出电压特性分析

图 10a 所示为激励频率(底座往复频率)分别在 $1 \mathrm{~Hz} 、 2 \mathrm{~Hz} 、 3 \mathrm{~Hz}$ 和 $4 \mathrm{~Hz}$ 下摩擦自激振动电压信号,

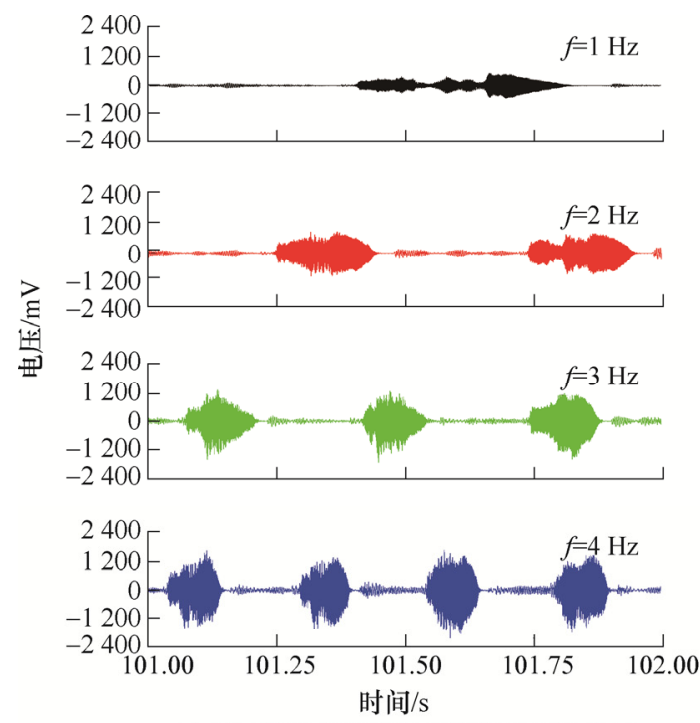

(a) 不同激励频率下的摩擦自激振动电压
其中, 往复行程均为 $10 \mathrm{~mm}$, 法向力均为 $20 \mathrm{~N}$ 。可 见试验结果均与理论计算结果相符, 摩擦自激振动 产生的电压信号幅值随着激励频率的增大而增大, 电压信号的高频振荡次数与激励频率相等。单个振 荡的时间随着激励频率增大而减小，但单位时间内 电压持续振荡的时间之和却增加, 说明压电输出能 量随着激振频率的增加而增加。

图 $10 \mathrm{~b}$ 所示为对应的四种不同激励频率下的碰 撞电压信号, 底座往复行程同样为 $10 \mathrm{~mm}$ 。从图 10 中可以看出, 输出电压呈现为脉冲信号, 压电悬臂 梁每撞击一次阻挡装置便输出一次电压脉冲, 因此 电压信号的脉冲次数与往复频率相等。当压电悬臂 梁撞上阻挡装置的一瞬间, 悬臂梁会产生较大变形, 其变形幅值远大于由高频摩擦自激振动所引起的悬 臂梁变形幅值，产生的电压幅值远高于摩擦自激振 动的电压幅值。然而, 该电压信号频率却远低于摩 擦自激振动的电压信号频率。由图 10 中可以看出, 随着底座往复频率的增大, 碰撞电压的幅值在增加, 其原因为底座往复频率上升后, 底座的运动速度加 快，压电悬臂梁与阻挡装置撞击的动能增加，压电 悬臂梁的变形增大。此外, 撞击频率上升后, 单个 脉冲信号的周期在缩短, 这是由于撞击的速度加快 后，悬臂梁的撞击和回弹周期相应缩短。
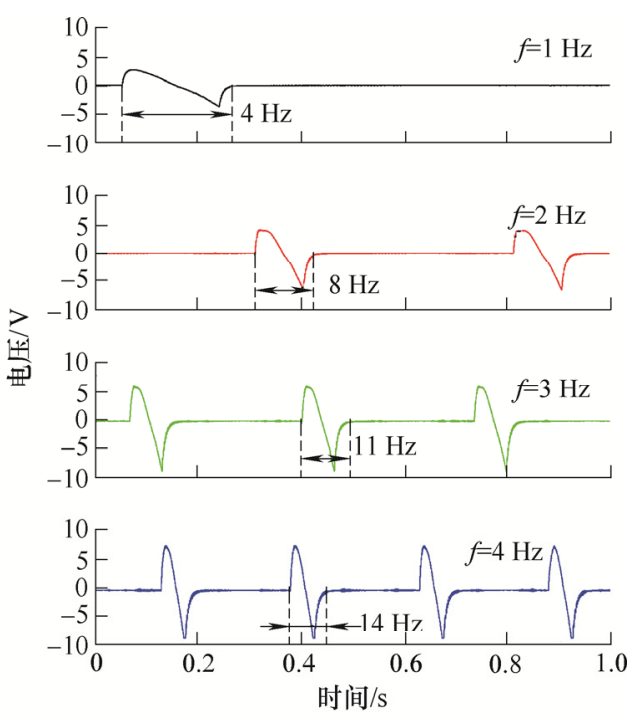

(b) 不同激励频率下的碰撞电压

图 10 两种能量回收方式的电压信号

对比摩擦自激振动电压和碰撞电压可以发现, 二者的区别主要体现在电压信号的频率和幅值方 面。摩擦自激振动电压幅值较小而频率很高, 碰撞 电压幅值很大但频率较低, 两种低频振动能量收集 方式优劣均较为明显。由式(6)可以得出, 压电陶瓷 的内部阻抗与压电电压频率呈反比, 因此摩擦自激
振动条件下压电陶瓷的内阻将会远小于碰撞条件下 压电陶瓷的内阻。结合式(8), 可以得出激励频率在 $1 \mathrm{~Hz}$ 及 $4 \mathrm{~Hz}$ 下摩擦自激振动和碰撞两种能量回收 方式的最优负载(外接负载等于电源内部阻抗)功率 曲线, 如图 11 所示。结果表明, 摩擦自激振动电压 的瞬时功率远大于碰撞电压的瞬时功率, 其原因在 
于电压的频率提升后, 降低了压电陶瓷内阻, 即使 摩擦自激振动电压信号幅值小于碰撞电压信号幅 值, 其最优负载下的瞬时功率仍远大于碰撞电压最 优负载下的瞬时功率。这说明通过摩擦自激振动的 方式能够将低频的激励转化为高频振动, 进而可以 提高压电悬臂梁的最优输出功率。
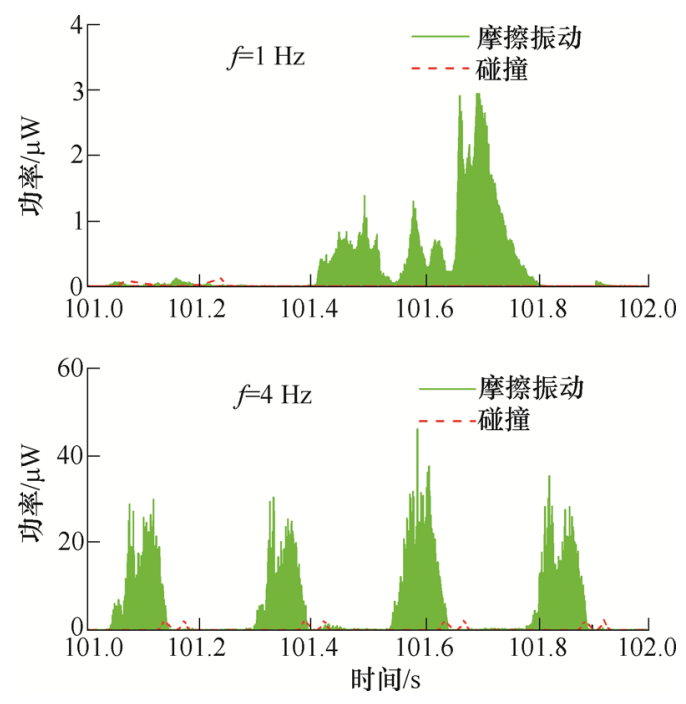

图 11 摩擦自激振动电压与碰撞电压功率曲线

对图 11 中的功率曲线进行积分, 得到一秒内压 电振子输出的能量, 结果如图 12 所示。随着频率的 增加, 两种能量收集方式收集到的能量都在增加。 其中, 高频的摩擦自激振动压电能量远高于低频的 碰撞压电能量, 这说明无论是瞬时功率, 还是单位 时间内的平均功率, 经过升频转换之后, 压电悬臂 梁的输出功效都得到了极大的提高。

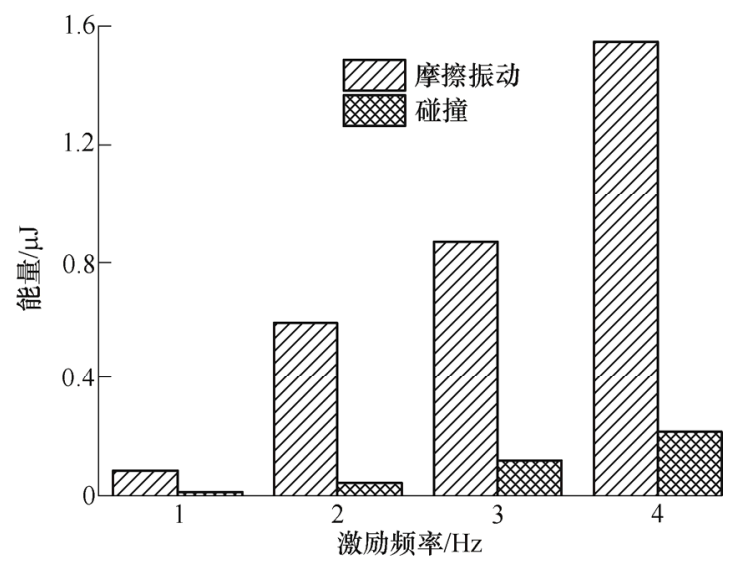

图 12 不同激励频率下两种能量回收方式的单位时间能量

\section{3 结论}

(1) 将低频振动转化为摩擦副之间的往复摩擦 可以激起摩擦系统的高频自激振动, 利用此高频振 动可收集到高频电压信号, 达到升频的效果。
(2) 数值模拟及试验结果均表明摩擦自激振动 电压信号幅值及其能量均随着激励频率的增加而 增大。

(3) 摩擦自激振动能量回收与碰撞式能量回收 对比试验结果表明, 摩擦自激振动电压幅值小于碰 撞电压幅值, 而频率却远高于碰撞电压。将低频激 励转化为摩擦系统的高频自激振动后, 振动频率得 到了提升, 压电陶瓷内阻降低, 压电的最优能量输 出得到了极大的提升。因此, 基于摩擦自激振动升 频效应的超低频振动能量收集方式十分适用于低频 的振动能量回收。

\section{参 考 文 献}

[1] BI S, HO C K, ZHANG R. Wireless powered communication: Opportunities and challenges[J]. IEEE Communication Magazine, 2015，53(4): 117-25.

[2] YANG $\mathrm{Y}, \mathrm{ZHOU} \mathrm{Y} \mathrm{S}, \mathrm{ZHANG} \mathrm{H}$, et al. A single-electrode based triboelectric nano generator as self-powered tracking system[J]. Advanced Materials, 2013, 25: 6594-6601.

[3] 陈小前, 袁建平, 姚雯, 等. 航天器在轨服务技术 $[\mathrm{M}]$. 北京: 中国宇航出版社, 2009.

CHEN Xiaoqian, YUAN Jianping, YAO Wen, et al. Spacecraft in-orbit service technology[M]. Beijing: China Astronautic Publishing House, 2009.

[4] 刘祥建, 陈仁文. 压电振动能量收集装置研究现状及发 展趋势 $[\mathrm{J}]$. 振动与冲击, 2012，31(36): 169-176.

LIU Xiangjian, CHEN Renwen. Review and prospect of micro-nano vibration energy harvesters [J]. Journal of Vibration and Shock, 2012, 31(36): 169-176.

[5] 元有超, 赵俊青, 张弛. 微纳振动能量收集器研究现状 与展望 $[\mathrm{J}]$. 机械工程学报, 2020, 56(13): 1-15.

QI Youchao, ZHAO Junqing, ZHANG Chi. Current situation and developing trend of piezoelectric vibration energy harvesters [J]. Journal of Mechanical Engineering, 2020, 56(13): 1-15.

[6] KIM H S, KIM J H, KIM J. A review of piezoelectric energy harvesting based on vibration[J]. International Journal of Precision Engineering and Manufacturing, 2011, 12: 1129-1141.

[7] 吴义鹏, 周圣鹏, 装进浩, 等. 用于超低频振动能收集 的压电弹簧摆结构设计与实现 $[\mathrm{J}]$. 振动工程学报, 2019, 32(5) : 750-756.

WU Yipeng, ZHOU Shengpeng, QIU Jinhao, et al. Design and implementation of a piezoelectric spring pendulum structure applied in ultra-low frequency vibration energy harvesting[J]. Journal of Vibration Engineering, 2019, 32(5): $750-756$. 
[8] 张思文, 吴九汇. 基于局部共振声子晶体结构的低频振 动能量回收研究 [J]. 固体力学学报, 2013, 34(4) : 333-341.

ZHANG Siwen, WU Jiuhui. Energy harvesting based on locally resonant phononic crystals for low frequency vibrations[J]. Chinese Journal of Solid Mechanics, 2013, 34(4): 333-341.

[9] WANG C, ZHANG Q C, WANG W. Low-frequency wideband vibration energy harvesting by using frequency up-conversion and quin-stable nonlinearity[J]. Journal of Sound and Vibration, 2017，399: 169-181.

[10] 李如春, 征琦, 林宇俊. 基于低频驱动的微压电能量收 集器的结构研究 [J]. 压电与声光, 2014(5): 735-738. LI Ruchun, ZHENG Qi, LIN Yujun. Study on the microstructure of piezoelectric energy harvester driven by low-frequency vibration[J]. Piezoelectrics and Acoustiooptics, 2014(5): 735-738.

[11] LIU H, LEE C, KOBAYASHI T, et al. Investigation of a MEMS piezoelectric energy harvester system with a frequency-widened-bandwidth mechanism introduced by mechanical stoppers[J]. Smart Materials and Structures 2012; $21: 035005$.

[12] 吴晔盛, 刘启, 曹杰, 等. 收集振动能的摩擦纳米发电 机设计与输出性能 [J]. 物理学报, 2019(19): 19-26. WU Yesheng, LIU Qi, CAO Jie, et al. Design and output performance of vibration energy harvesting triboelectric nanogenerator[J]. Acta Phys. Sin., 2019(19): 19-26.

[13] LIU Huicong, LEE Chengkuo, TAKESHI K, et al. Piezoelectric MEMS-based wideband energy harvesting systems using a frequency-up-conversion cantilever
stopper[J]. Sensors and Actuators A: Physical, 2012, 186: 242-248.

[14] TONAZZI D, MASSI F, BAILLET L, et al. Interaction between contact behavior and vibrational response for dry contact system[J]. Mechanical Systems and Signal Processing, 2018, 110: 110-121.

[15] WANG D W, MO J L, WANG X F, et al. Experimental and numerical investigations of the piezoelectric energy harvesting via friction-induced vibration[J]. Energy Conversion and Management, 2018, 171: 1134-1149.

[16] TADOKORO C, MATSUMOTO A, NAGAMINE T, et al. Piezoelectric power generation using friction-induced vibration[J]. Smart Materials and Structures, 2017，26: 065012 .

[17] GREEN P L, WORDEN K, SIMS N D. On the identification and modelling of friction in a randomly excited energy harvester[J]. Journal of Sound and Vibration, 2013, 332: 4696-4708.

[18] 王东伟, 莫继良, 王正国, 等. 沟槽织构化表面影响摩 擦振动噪声机理 [J]. 机械工程学报, 2013, 49(23): 112-116.

WANG Dongwei, MO Jiliang, WANG Zhengguo, et al. Mechanism of the effect of Groove-textured surface on the friction vibration and noise[J]. Journal of Mechanical Engineering, 2013, 49(23): 112-116.

作者简介: 陈伟, 男, 1996 年出生。主要研究方向为摩擦振动能量收集。 E-mail: 2847509730@qq.com 莫继良(通信作者), 男, 1982 年出生, 博士, 研究员, 博士研究生导师。 主要研究方向为摩擦振动噪声、摩擦学及表面工程。

E-mail: jlmo@swjtu.cn 